\title{
The role of hydrogen in Alzheimer's disease
}

\author{
Xin Tan ${ }^{1, *}$, Fang Shen ${ }^{2, *}$, Wan-Li Dong ${ }^{1,{ }^{*}}$, Yi Yang ${ }^{1,}{ }^{\star}$, Gang Chen ${ }^{2}$ \\ 1 Department of Neurology, the First Affiliated Hospital of Soochow University, Suzhou, Jiangsu Province, China \\ 2 Department of Neurosurgery \& Brain and Nerve Research Laboratory, the First Affiliated Hospital of Soochow University, Suzhou, Jiangsu \\ Province, China
}

\#These authors contributed equally to this work.

*Correspondence to: Wan-Li Dong, dwlsz8@163.com; Yi Yang, 447378224@qq.com.

orcid: 0000-0001-7896-9485 (Yi Yang)

\section{Abstract}

Alzheimer's disease is one of the most common neurodegenerative diseases in the elderly. It is often manifested as learning and memory impairment, cognitive function decline, normal social and emotional disorders. However, for this high-risk common disease, there is currently no effective treatment, which has plagued many clinicians. As a new type of medical therapeutic gas, hydrogen has attracted much attention recently. As a recognized reducing gas, hydrogen has shown great anti-oxidative stress and anti-inflammatory effect in many cerebral disease models. It can ameliorate neuronal damage, maintain the number of neurons, prolong the lifespan of neurons, and ultimately inhibit disease progression. Therefore, the role and mechanism of hydrogen in the pathological process of Alzheimer's disease will be discussed in this paper.

Key words: hydrogen; Alzheimer's disease; experimental research; underlying mechanism; therapeutic implications; neuroprotection; antiinflammation; anti-oxidative stress

doi: $10.4103 / 2045-9912.248270$

How to cite this article: Tan X, Shen F, Dong WL, Yang Y, Chen G. The role of hydrogen in Alzheimer's disease. Med Gas Res. 2018;8(4):176180 .

\section{INTRODUCTION}

As is known to all, neurodegenerative diseases lead to the motor and cognitive disorder gradually. The characteristics of the neurodegenerative diseases is to reduce the number of cells in the brain and spinal cord, which are not renewable generally. ${ }^{1}$ Alzheimer's disease (AD) is known to be the most common neurodegenerative disease. As its characteristics, the abnormal aggregation of $\beta$-amyloid $(\mathrm{A} \beta)$ and tau can be discovered. ${ }^{2}$ Numerous studies on AD have been widely carried out in recent years. Moreover, researches of hydrogen therapy on neurodegenerative diseases have become a hotspot.

As the smallest gas molecule, molecular hydrogen is made of two protons and two electrons. In the past, it was considered to be physiologically inert in mammalian cells. In 1975, hydrogen was firstly discovered to show therapeutic effects in a skin squamous carcinoma mouse model. ${ }^{3}$ In 2007, it was reported to show antioxidant properties by reducing hydroxyl radicals $(\bullet \mathrm{OH})$, which aroused worldwide concern. ${ }^{4}$ A large number of researches have been carried out to prove that hydrogen plays favorable effect in gastric diseases, ${ }^{5}$ eye injury, ${ }^{6,7}$ otology diseases, ${ }^{8}$ oral diseases,${ }^{9}$ allergic rhinitis, ${ }^{10}$ acute pancreatitis, ${ }^{11}$ urinal system diseases, ${ }^{12}$ skin disease, ${ }^{13}$ hematopoietic system disorders, ${ }^{14,15}$ mental diseases, ${ }^{16}$ neurodegenerative diseases, ${ }^{17}$ traumatic brain injury, ${ }^{18}$ spinal cord ischemia/reperfusion injury, ${ }^{19}$ and ischemic brain injury. ${ }^{20-22}$ Hydrogen has been used in the study of so many disease models, so its safety also has received worldwide attention. The ignition point of hydrogen is $574^{\circ} \mathrm{C}$, and it explodes with oxygen in the hydrogen concentration range of $4-75 \%$ (vol/ vol). ${ }^{23}$ Therefore, less than $4 \%$ of low concentration hydrogen which has demonstrated great efficacy is safe in researches and applications. ${ }^{24}$ On the one hand, compared with other medical gases, hydrogen has a more prominent advantage in toxicity.
Even at high concentrations, hydrogen is still non-toxic ${ }^{25}$ and has been used in diving applications. ${ }^{26}$ Studies had showed that hydrogen inhalation did not have obvious adverse effects ${ }^{4}$ and had no influence on blood pressure and other blood parameters such as $\mathrm{pH}$, temperature. ${ }^{27}$ This is consistent with the results of recent clinical trials in patients with cerebral infarction ${ }^{28}$ and post-cardiac arrest syndrome. ${ }^{29}$ Moreover, solubilized hydrogen such as hydrogen-rich water was recognized to be safe and easily administered in many researches. ${ }^{30-32}$ A clinical trial conducted by Nagatani et al. ${ }^{33}$ showed that intravenous solution of hydrogen-rich water was safe for patients who were suffering acute cerebral infarction, including those treated with tissue plasminogen activator. On the other hand, compared with other antioxidants, hydrogen showed less side effects since it only reduced $\bullet \mathrm{OH} .{ }^{4}$ In summary, the safety and nontoxicity of hydrogen have been confirmed in many studies. In our review, we focus on discussing the role of hydrogen in $\mathrm{AD}$ and summarizing possible molecular mechanisms.

\section{MeCHANISMS OF THE HydROGen THERAPY IN ALZHEIMER'S DISEASE}

$\mathrm{AD}$ is the most common neurodegenerative diseases which result in dementia. ${ }^{34,35} \mathrm{AD}$ patients often show decreased ability of learning and memory, impaired cognitive function, normal social and emotional disorders. ${ }^{36}$ Inflammation and oxidative stress are recognized as the main causes of $\mathrm{AD},{ }^{37}$ Parkinson's disease, ${ }^{38}$ and other neurodegenerative diseases. Pathologically the deposition of $A \beta$ which results in neuritic plaques and the hyperphosphorylated tau protein are prominent features in $\mathrm{AD} .^{39}$ The overproduction of $\mathrm{A} \beta$ leads to dysfunction of mitochondrial complexes, which contribute to the overproduction of reactive oxygen species (ROS) and the depletion of adenosine triphosphate (ATP). ${ }^{40}$ ATP is important 
to neurotransmission and axonal transport, and it helps maintain the function of the ion channel and maintains the balance of ions inside and outside the cell..$^{40}$ So the depletion of ATP is the cause of mitochondrial dynamics damage. ${ }^{40}$ Moreover, an increase in the amount of ROS causes a change in the pore size of the mitochondrial permeability transition pore, causing calcium ions to flow into the mitochondria, which in turn further exacerbates mitochondrial damage. ${ }^{40}$ And ROS can also affect the function of the membrane to cause lipid peroxidation, promote cell apoptosis, and reduce the number of neurons. ${ }^{40}$ Mitochondrial damage also caused by tau protein, which results in energy dysfunction, ROS production, and ultimately to affect synaptic function. ${ }^{41,42}$ And ROS in turn accelerates tau hyperphosphorylation..$^{40}$ In summary, disorder of cholinergic function, amyloid cascade, oxidative stress, inflammation, excitotoxicity and steroid hormone deficiencies have been recognized to be the pathogenic mechanisms for $\mathrm{AD}{ }^{43}$

In addition, hydrogen molecules such as hydrogen-rich water, hydrogen-rich saline or hydrogen inhalation exhibit anti-inflammatory and anti-oxidant effects in many studies. ${ }^{44,45}$ In many disease models, many pro-inflammatory cytokines are down-regulated such as nuclear factor- $\kappa \mathrm{B},{ }^{46}$ interleukin- $1 \beta$, interleukin-6, interleukin-10, tumor necrosis factor- $\alpha$ (TNF- $\alpha$ ), chemokine (C-C motif) ligand 2 , interferon- $\gamma$, and intercellular adhesion molecule- 1 , etc. ${ }^{47}$ to exert anti-inflammatory effects by given hydrogen. ${ }^{1}$ This effect also exists in the AD models. ${ }^{48}$ In AD transgenic mouse model, the decline of nucleotide-binding domain leucin-rich repeat and pyrin domain-containing protein 3 (NLRP3) was proved to inhibit memory impairment and $\mathrm{A} \beta$ deposition. ${ }^{49} \mathrm{~A}$ current investigation reported that hydrogen could inhibit the activation of NLRP3 inflammasome in AD brains. ${ }^{50}$

Hydrogen can also stimulate energy metabolism to reduce neuronal damage. For example, it could up-regulate the expression of fibroblast growth factor $21^{51}$ and ghrelin. ${ }^{52}$ Lin et al. ${ }^{53}$ found that hydrogen-rich water can stimulate AMPK-Sirt1$\mathrm{FoxO}_{3}$ a pathway which could play a role in anti-oxidative stress, diminishing mitochondrial damage and acting as a neuroprotective agent, and neutralize ROS induced by A $\beta$. Sirt1 could also induce autophagy, which plays a neuroprotective role in many neurodegenerative diseases. ${ }^{54}$ So hydrogen can also protect cells by promoting autophagy in AD. And as is known to all, autophagy is an indispensable process to maintain cell homeostasis. ${ }^{55}$ As members of the mitogen-activated protein kinase, phospho-p38 and c-Jun $\mathrm{NH}_{2}$-terminal kinase (JNK) participate in regulating cell survival. ${ }^{56,57}$ Henderson et al. ${ }^{58}$ reported that activated by oxidative stress $\mathrm{P} 38 \mathrm{~K}$ enhanced Bax phosphorylation and its translocation into mitochondria which led to apoptosis and neurodegeneration in AD brains. In many models, the results showed that that hydrogen water could suppress the activation of phospho-p38 and JNK. ${ }^{48,59,60}$ This is consistent with other findings that molecular hydrogen can reduce neuronal apoptosis by inhibiting ROS-activated caspase signaling and protecting mitochondria. ${ }^{61}$ In this regard, in $\mathrm{AD}$, hydrogen treatment has anti-apoptotic effects.

Currently, Hou et al. ${ }^{50}$ reported that hydrogen-rich water could improve cognition function in female transgenic $\mathrm{AD}$ mice by reducing the decline in brain estrogen levels, estrogen receptor (ER) $\beta$, and the expression of brain-derived neuro- trophic factor (BDNF), but not in males without affecting $\beta$-amyloid precursor protein processing and $A \beta$ clearance. Furthermore, the suppression of inflammatory responses and oxidative stress was more pronounced in female AD mice than the males. ${ }^{50}$ This suggests that hydrogen can also participate in the pathogenesis of AD by affecting the estrogen-ER $\beta$-BDNF signaling pathway. And estrogen can inhibit AD progression and neuronal damage by mitogen-activated protein kinase ${ }^{62}$ and protein kinase $\mathrm{C}$ signaling pathway. ${ }^{63}$ At the same time, BDNF and tyrosine kinase receptor B is thought to up-regulate the expression of genes which are associated with the differentiation of neuron, neuronal survival. Finally, it will improve synaptic plasticity and enhance learning and memory ability. ${ }^{64}$ Moreover, the estrogen-ER $\beta$-BDNF signaling pathway was associated with antioxidative and anti-inflammatory effects in AD. ${ }^{65} 17 \beta$-oestradiol-induced signaling improved mitochondrial function which was related to ATP generation and oxidative phosphorylation. ${ }^{66}$ The activation of ER $\beta$ signaling was also involved in ROS scavenging in pathological AD prevention. ${ }^{67}$

Therefore, anti-inflammatory, anti-oxidative stress, antiapoptotic and the regulation of both autophagy and hormone signaling pathway are the main mechanisms of action of hydrogen.

\section{Studies of Hydrogen Therapy in Alzheimer's DISEASE}

Numerous studies have been carried out. Nagata et al. ${ }^{68}$ reported that consumption of hydrogen water could prevent the decline of cognition, and maintain the proliferation of neural progenitors, and inhibit oxidative stress after chronic restraint stress in a mouse model of dementia. They observed the rise of malondialdehyde and 4-hydroxy-2-nonenal, which were recognized as oxidative stress markers enhanced by chronic restraint stress, was suppressed by using hydrogen water. ${ }^{68} \mathrm{At}$ the same time, hydrogen water restored the decrease in the number of proliferating cells in dentate gyrus after restraint stress. ${ }^{68}$ Neurogenesis in the adult hippocampus keeps changing, which plays an important role in learning, memory and hippocampal plasticity. ${ }^{69,70}$ The current study showed that cognitive impairments, pathological tau aggregation which were the characteristics of AD can be induced by the reduction of hippocampal neurogenesis. ${ }^{69}$ This indirectly demonstrated that the effectiveness of hydrogen in the treatment of AD. In 2010, Li et al. ${ }^{71}$ found that hydrogen-rich saline could reduce learning and memory impairments and neural inflammation which were induced by $A \beta$ in rats. They observed that hydrogen saline greatly improved learning memory and long-term potentiation (LTP), a form of synaptic plasticity related to learning and memory closely. ${ }^{71}$ LTP was recognized to be blocked by $A \beta$ peptide oligomers rapidly and obviously. ${ }^{71}$ Moreover, hydrogen-rich saline suppressed lipid peroxidation products, inflammatory factor like interleukin- 6 and TNF- $\alpha$, and the activation of astrocytes. ${ }^{71}$ TNF- $\alpha$ also participated in the inhibition of LTP induced by A $\beta .^{71}$ In the following year, the same team published that the protective effect of hydrogenrich saline may be due to inhibition of the activation of JNK and NF- $\mathrm{KB} .{ }^{48}$ Another study showed that drinking hydrogen water for 30 days could exhibit the age-related impairment of 
learning ability and memory in senescence-accelerated mouse prone 8 strain (SAMP8). ${ }^{72}$ Additionally, senescence-accelerated prone mouse 8 showed the alleviation of the reduction of neurons in the hippocampus after being treated with hydrogen water for 18 weeks. ${ }^{72}$ A similar study was discovered in 2015 by Kiyomi et al..$^{73}$ They used transgenic mice (DAL101) which lacked the activity of aldehyde dehydrogenase 2 as a dementia model. And they found that hydrogen-water could decrease oxidative stress and prevent the decline in cognition, learning and memory impairment and weaken neurodegeneration and the mean of lifespan of mice was extended. Moreover, they conducted a randomized clinical study which showed that hydrogen can greatly improve the cognition in the apolipoprotein E4 genotype carriers. ${ }^{73}$ Recently, more and more evidence has shown that apolipoprotein E participates in anti-inflammatory, antioxidative and antiapoptotic effects during the process of brain injuries. ${ }^{74}$ However, apolipoprotein E4 is thought to play a role in promoting oxidation, tau phosphorylation and the production of $A \beta$ in the pathological process of AD. ${ }^{40} \mathrm{All}$ experimental studies are shown in Table 1. Numerous studies are still underway, and clinical trials are being carried out gradually throughout the country.

\section{Prospects}

From an epidemiological point of view, women tend to have more extensive manifestations of dementia, and they experience more severe cognitive degeneration than men during $\mathrm{AD}$ progression..$^{75}$ And the loss of ovarian hormones is considered to be susceptible to AD in menopausal women. ${ }^{76}$ Whereas hydrogen-rich water could reduce the decline in brain estrogen levels in females. ${ }^{50}$ Therefore, hydrogen therapy for elderly women with AD may have a better effect. However, the specific mechanism about how hydrogen molecules inhibit the loss of estrogen remains unclear and further research is needed. On the one hand, Sirt1-FoxO3A axis might represent a new target for $\mathrm{AD}$ treatment. On the other hand in the pathology of $\mathrm{AD}$, mitochondrial dysfunction is a non-negligible part, which may be another direction for the diagnosis and treatment of $\mathrm{AD}$ in the future. Currently, cathepsin $\mathrm{B}$ has been found that it links to NLRP3 inflammasome activation in AD ${ }^{77}$ So whether cathepsin B is a target for hydrogen therapy also requires follow-up research.

\section{ConcLusion}

Hydrogen is a common non-toxic and safe medical gas, and its beneficial effects on AD have been confirmed in many studies, mainly through anti-inflammatory, anti-oxidative stress, antiapoptotic and the regulation of both autophagy and hormone signaling pathway. Small-scale clinical studies have begun on the role of hydrogen in $\mathrm{AD}$, so hydrogen is a new type of medical gas with great development prospects and we expect larger-scale clinical studies to be implemented.

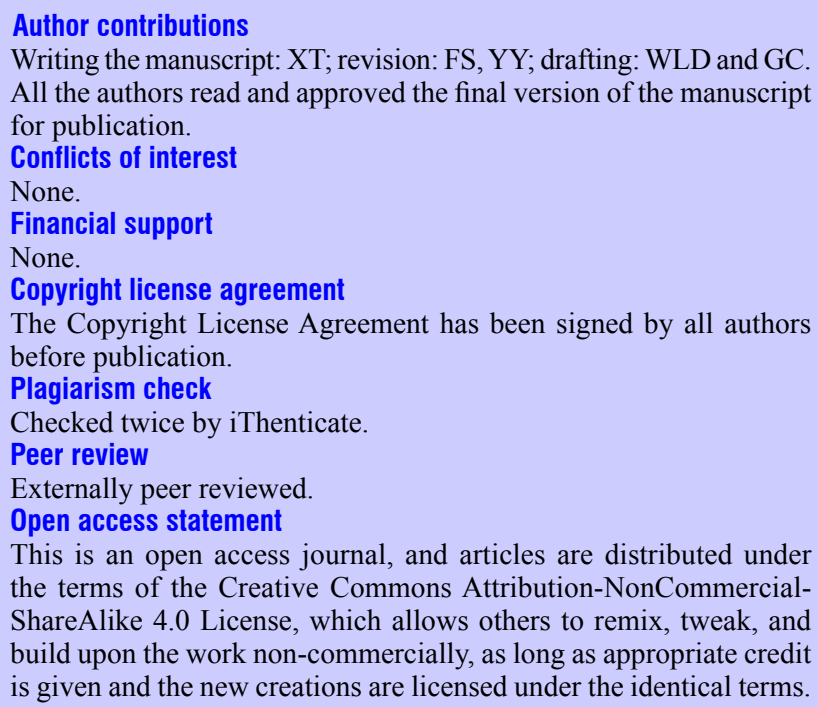

Writing the manuscript: XT; revision: FS, YY; drafting: WLD and GC. All the authors read and approved the final version of the manuscript for publication.

Conflicts of interest

None.

Financial support

None.

Copyright license agreement

The Copyright License Agreement has been signed by all authors before publication.

Plagiarism check

Checked twice by iThenticate.

Peer review

Externally peer reviewed.

Open access statement

This is an open access journal, and articles are distributed under the terms of the Creative Commons Attribution-NonCommercialShareAlike 4.0 License, which allows others to remix, tweak, and build upon the work non-commercially, as long as appropriate credit is given and the new creations are licensed under the identical terms.

\section{REFERENCES}

1. Kovacs GG. Concepts and classification of neurodegenerative diseases. Handb Clin Neurol. 2017;145:301-307.

2. Jucker M, Walker LC. Pathogenic protein seeding in Alzheimer disease and other neurodegenerative disorders. Ann Neurol. 2011;70:532-540.

3. Dole M, Wilson FR, Fife WP. Hyperbaric hydrogen therapy: a possible treatment for cancer. Science. 1975;190:152-154.

Table 1: Experimental studies of hydrogen in AD

\begin{tabular}{|c|c|c|c|}
\hline Author & Animals/cells & Model & Results \\
\hline Hou et al. ${ }^{50}$ & Mice & $\mathrm{AD}$ & $\begin{array}{l}\text { Hydrogen-rich water inhibit NLRP3, and weaken the oestrogen-ER } \beta \text {-BDNF } \\
\text { signalling pathway. }\end{array}$ \\
\hline Lin et al. ${ }^{53}$ & $\begin{array}{l}\text { Human } \\
\text { neuroblastoma } \\
\text { SK-N-MC cells }\end{array}$ & $\mathrm{AD}$ & $\begin{array}{l}\text { Hydrogen-rich water unregulated AMPK-Sirt1-FoxO3a pathway and } \\
\text { neutralize excessive ROS to protect neuron. }\end{array}$ \\
\hline $\begin{array}{l}\text { Nagata et } \\
\text { al. }{ }^{68}\end{array}$ & Mice & $\begin{array}{l}\text { Dementia induced by chronic } \\
\text { physical restraint stress }\end{array}$ & $\begin{array}{l}\text { Molecular hydrogen inhibited the stress-induced learning and memory } \\
\text { impairments. }\end{array}$ \\
\hline Li et al. ${ }^{71}$ & Rats & $\mathrm{AD}$ & $\begin{array}{l}\text { Hydrogen-rich saline improved memory function by inhibition of oxidative } \\
\text { stress and reduction of interleukin- } 6 \text { and TNF- } \alpha \text { and the activation of } \\
\text { astrocytes. }\end{array}$ \\
\hline Gu et al. ${ }^{72}$ & Mice & $\mathrm{AD}$ & Hydrogen water ameliorated neurodegeneration in hippocampus. \\
\hline 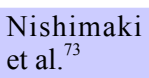 & Mice & Dementia & $\begin{array}{l}\text { Molecular hydrogen improve the cognition in the apolipoprotein E4 geno-type } \\
\text { carriers. }\end{array}$ \\
\hline
\end{tabular}

Note: AD: Alzheimer's disease; TNF-a: tumor necrosis factor-a; NLRP3: nucleotide-binding domain leucin-rich repeat and pyrin domain-containing protein 3; ROS: reactive oxygen species; ER: estrogen receptor; BDNF: brain-derived neurotrophic factor. 
4. Ohsawa I, Ishikawa M, Takahashi K, et al. Hydrogen acts as a therapeutic antioxidant by selectively reducing cytotoxic oxygen radicals. Nat Med. 2007;13:688-694.

5. Zhang JY, Wu QF, Wan Y, et al. Protective role of hydrogen-rich water on aspirin-induced gastric mucosal damage in rats. World $J$ Gastroenterol. 2014;20:1614-1622.

6. Cejka C, Kossl J, Hermankova B, Holan V, Cejkova J. Molecular hydrogen effectively heals alkali-injured cornea via suppression of oxidative stress. Oxid Med Cell Longev. 2017;2017:8906027.

7. Yan W, Chen T, Long P, et al. Effects of post-treatment hydrogen gas inhalation on uveitis induced by endotoxin in rats. Med Sci Monit. 2018;24:3840-3847.

8. Chen L, Han M, Lu Y, et al. Molecular mechanisms underlying the protective effects of hydrogen-saturated saline on noise-induced hearing loss. Acta Otolaryngol. 2017;137:1063-1068.

9. Tamaki N, Orihuela-Campos RC, Fukui M, Ito HO. Hydrogen-rich water intake accelerates oral palatal wound healing via activation of the Nrf2/antioxidant defense pathways in a rat model. Oxid Med Cell Longev. 2016;2016:5679040.

10. Xu FF, Yu SQ, Zhao CL, et al. Effect of hydrogen-rich saline on the CD4(+) CD25(+) Foxp3(+) Treg cells of allergic rhinitis guinea pigs model. Zhonghua Er Bi Yan Hou Tou Jing Wai Ke Za Zhi. 2017;52:506-511.

11. Han B, Zhou H, Jia G, et al. MAPKs and Hsc70 are critical to the protective effect of molecular hydrogen during the early phase of acute pancreatitis. FEBS J. 2016;283:738-756.

12. Miyazaki N, Yamaguchi O, Nomiya M, Aikawa K, Kimura J. Preventive effect of hydrogen water on the development of detrusor overactivity in a rat model of bladder outlet obstruction. $J$ Urol. 2016;195:780-787.

13. Watanabe S, Fujita M, Ishihara M, et al. Protective effect of inhalation of hydrogen gas on radiation-induced dermatitis and skin injury in rats. J Radiat Res. 2014;55:1107-1113.

14. Zhang J, Xue X, Han X, et al. Hydrogen-rich water ameliorates total body irradiation-induced hematopoietic stem cell injury by reducing hydroxyl radical. Oxid Med Cell Longev. 2017;2017:8241678.

15. Guo J, Zhao D, Lei X, et al. Protective effects of hydrogen against low-dose long-term radiation-induced damage to the behavioral performances, hematopoietic system, genital system, and splenic lymphocytes in mice. Oxid Med Cell Longev. 2016;2016:1947819.

16. Wen D, Zhao P, Hui R, et al. Hydrogen-rich saline attenuates anxiety-like behaviors in morphine-withdrawn mice. Neuropharmacology. 2017;118:199-208.

17. Yoshii Y, Inoue T, Uemura Y, et al. Complexity of stomach-brain interaction induced by molecular hydrogen in parkinson's disease model mice. Neurochem Res. 2017;42:2658-2665.

18. Tian R, Hou Z, Hao S, et al. Hydrogen-rich water attenuates brain damage and inflammation after traumatic brain injury in rats. Brain Res. 2016;1637:1-13.

19. Zhou L, Wang X, Xue W, et al. Beneficial effects of hydrogen-rich saline against spinal cord ischemia-reperfusion injury in rabbits. Brain Res. 2013;1517:150-160.

20. Han L, Tian R, Yan H, et al. Hydrogen-rich water protects against ischemic brain injury in rats by regulating calcium buffering proteins. Brain Res. 2015;1615:129-138.

21. Yuan J, Wang D, Liu Y, et al. Hydrogen-rich water attenuates oxidative stress in rats with traumatic brain injury via $\mathrm{Nrf2}$ pathway. $J$ Surg Res. 2018;228:238-246.

22. Chan SJ, Wong PT. Reprint of hydrogen sulfide in stroke: Protective or deleterious? Neurochem Int. 2017;107:78-87.

23. Ohta S. Recent progress toward hydrogen medicine: potential of molecular hydrogen for preventive and therapeutic applications. Curr Pharm Des. 2011;17:2241-2252.

24. Hayashida K, Sano M, Ohsawa I, et al. Inhalation of hydrogen gas reduces infarct size in the rat model of myocardial ischemia-reperfusion injury. Biochem Biophys Res Commun. 2008;373:30-35.

25. Abraini JH, Gardette-Chauffour MC, Martinez E, Rostain JC, Lemaire C. Psychophysiological reactions in humans during an open sea dive to $500 \mathrm{~m}$ with a hydrogen-helium-oxygen mixture. $J$ Appl Physiol (1985). 1994;76:1113-1118.

26. Fontanari $\mathrm{P}$, Badier $\mathrm{M}$, Guillot $\mathrm{C}$, et al. Changes in maximal performance of inspiratory and skeletal muscles during and after the 7.1-MPa Hydra 10 record human dive. Eur J Appl Physiol. 2000;81:325-328.
27. Ono H, Nishijima Y, Adachi N, et al. A basic study on molecular hydrogen $\left(\mathrm{H}_{2}\right)$ inhalation in acute cerebral ischemia patients for safety check with physiological parameters and measurement of blood $\mathrm{H}_{2}$ level. Med Gas Res. 2012;2:21.

28. Ono H, Nishijima Y, Ohta S, et al. Hydrogen gas inhalation treatment in acute cerebral infarction: a randomized controlled clinical study on safety and neuroprotection. J Stroke Cerebrovasc Dis. 2017;26:2587-2594.

29. Tamura T, Hayashida K, Sano M, et al. Feasibility and safety of hydrogen gas inhalation for post-cardiac arrest syndrome- first-inhuman pilot study. Circ J. 2016;80:1870-1873.

30. Koyama Y, Taura K, Hatano E, et al. Effects of oral intake of hydrogen water on liver fibrogenesis in mice. Hepatol Res. 2014;44:663-677.

31. Ara J, Fadriquela A, Ahmed MF, et al. Hydrogen water drinking exerts antifatigue effects in chronic forced swimming mice via antioxidative and anti-inflammatory activities. Biomed Res Int. 2018;2018:2571269.

32. Jin L, Yu SQ, Zhang X, et al. Clinical study of hydrogen-rich saline in the treatment of moderate to severe allergic rhinitis. Lin Chung Er Bi Yan Hou Tou Jing Wai Ke Za Zhi. 2018;32:493-496.

33. Nagatani K, Nawashiro H, Takeuchi S, et al. Safety of intravenous administration of hydrogen-enriched fluid in patients with acute cerebral ischemia: initial clinical studies. Med Gas Res. 2013;3:13.

34. Huang WJ, Zhang X, Chen WW. Role of oxidative stress in Alzheimer's disease. Biomed Rep. 2016;4:519-522.

35. Jembrek MJ, Hof PR, Šimić G. Ceramides in Alzheimer's disease: key mediators of neuronal apoptosis induced by oxidative stress and A $\beta$ accumulation. Oxid Med Cell Longev. 2015;2015:346783.

36. Sona A, Ellis KA, Ames D. Rapid cognitive decline in Alzheimer's disease: a literature review. Int Rev Psychiatry. 2013;25:650-658.

37. Wang X, Wang W, Li L, Perry G, Lee HG, Zhu X. Oxidative stress and mitochondrial dysfunction in Alzheimer's disease. Biochim Biophys Acta. 2014;1842:1240-1247.

38. Hald A, Lotharius J. Oxidative stress and inflammation in Parkinson's disease: is there a causal link? Exp Neurol. 2005;193:279290.

39. Luquecontreras D, Carvajal K, Toralrios D, Francobocanegra D, Campospeña V. Oxidative stress and metabolic syndrome: cause or consequence of Alzheimer's disease? Oxid Med Cell Longev. 2015;2014:497802.

40. Luque-Contreras D, Carvajal K, Toral-Rios D. Oxidative stress and metabolic syndrome: cause or consequence of Alzheimer's disease? Oxid Med Cell Longev. 2014;2014:497802.

41. Rapoport SI. Coupled reductions in brain oxidative phosphorylation and synaptic function can be quantified and staged in the course of Alzheimer disease. Neurotox Res. 2003;5:385.

42. Lasagnareeves CA, Castillocarranza DL, Sengupta U, Clos AL, Jackson GR, Kayed R. Tau oligomers impair memory and induce synaptic and mitochondrial dysfunction in wild-type mice. $\mathrm{Mol}$ Neurodegener. 2011;6:39.

43. Shah RS, Lee HG, Xiongwei Z, Perry G, Smith MA, Castellani RJ. Current approaches in the treatment of Alzheimer's disease. Biomed Pharmacother. 2008;62:199-207.

44. Cui Y, Zhang H, Ji M, et al. Hydrogen-rich saline attenuates neuronal ischemia--reperfusion injury by protecting mitochondrial function in rats. J Surg Res. 2014;192:564-572.

45. Mano Y, Kotani T, Ito M, et al. Maternal molecular hydrogen administration ameliorates rat fetal hippocampal damage caused by in utero ischemia-reperfusion. Free Radic Biol Med. 2014;69:324330.

46. Chen CH, Manaenko A, Zhan Y, et al. Hydrogen gas reduced acute hyperglycemia-enhanced hemorrhagic transformation in a focal ischemia rat model. Neuroscience. 2010;169:402-414.

47. Ohta S. Molecular hydrogen as a preventive and therapeutic medical gas: initiation, development and potential of hydrogen medicine. Pharmacol Ther. 2014;144:1-11.

48. Wang C, Li J, Liu Q, et al. Hydrogen-rich saline reduces oxidative stress and inflammation by inhibit of JNK and NF-kappaB activation in a rat model of amyloid-beta-induced Alzheimer's disease. Neurosci Lett. 2011;491:127-132.

49. Tan MS, Yu JT, Jiang T, Zhu XC, Tan L. The NLRP3 inflammasome in Alzheimer's disease. Mol Neurobiol. 2013;48:875-882. 
50. Hou C, Peng Y, Qin C, Fan F, Liu J, Long J. Hydrogen-rich water improves cognitive impairment gender-dependently in APP/PS1 mice without affecting Abeta clearance. Free Radic Res. 2018:112 .

51. Kamimura N, Nishimaki K, Ohsawa I, Ohta S. Molecular hydrogen improves obesity and diabetes by inducing hepatic FGF21 and stimulating energy metabolism in $\mathrm{db} / \mathrm{db}$ mice. Obesity (Silver Spring). 2011;19:1396-1403.

52. Matsumoto A, Yamafuji M, Tachibana T, Nakabeppu Y, Noda M, Nakaya H. Oral 'hydrogen water' induces neuroprotective ghrelin secretion in mice. Sci Rep. 2013;3:3273.

53. Lin CL, Huang WN, Li HH, et al. Hydrogen-rich water attenuates amyloid beta-induced cytotoxicity through upregulation of Sirt1FoxO3a by stimulation of AMP-activated protein kinase in SK-NMC cells. Chem Biol Interact. 2015;240:12-21.

54. Yao H, Zhao D, Khan SH, Yang L. Role of autophagy in prion protein-induced neurodegenerative diseases. Acta Biochim Biophys Sin (Shanghai). 2013;45:494-502.

55. Riahi Y, Wikstrom JD, Bachar-Wikstrom E, et al. Autophagy is a major regulator of beta cell insulin homeostasis. Diabetologia. 2016;59:1480-1491.

56. Xia Z, Dickens M, Raingeaud J, Davis RJ, Greenberg ME. Opposing effects of ERK and JNK-p38 MAP kinases on apoptosis. Science. 1995;270:1326-1331.

57. Rafieipour F, Hadipour E, Emami SA, Asili J, Tayarani-Najaran Z. Safranal protects against beta-amyloid peptide-induced cell toxicity in PC12 cells via MAPK and PI3 K pathways. Metab Brain Dis. 2018. doi: 10.1007/s11011-018-0329-9.

58. Henderson LE, Abdelmegeed MA, Yoo SH, et al. Enhanced phosphorylation of Bax and its translocation into mitochondria in the brains of individuals affiliated with Alzheimer's disease. Open Neurol J. 2017;11:48-58

59. Zhai Y, Zhou X, Dai Q, Fan Y, Huang X. Hydrogen-rich saline ameliorates lung injury associated with cecal ligation and puncture-induced sepsis in rats. Exp Mol Pathol. 2015;98:268-276.

60. Liu X, Chen Z, Mao N, Xie Y. The protective of hydrogen on stressinduced gastric ulceration. Int Immunopharmacol. 2012;13:197203.

61. Luo Y, Yang X, Zhao S, et al. Hydrogen sulfide prevents OGD/Rinduced apoptosis via improving mitochondrial dysfunction and suppressing an ROS-mediated caspase-3 pathway in cortical neurons. Neurochem Int. 2013;63:826-831.

62. Fitzpatrick JL, Mize AL, Wade CB, Harris JA, Shapiro RA, Dorsa DM. Estrogen-mediated neuroprotection against beta-amyloid toxicity requires expression of estrogen receptor alpha or beta and activation of the MAPK pathway. J Neurochem. 2002;82:674-682.

63. Cordey M, Gundimeda U, Gopalakrishna R, Pike CJ. Estrogen activates protein kinase $\mathrm{C}$ in neurons: role in neuroprotection. $J$ Neurochem. 2003;84:1340-1348.

64. Fukuda M, Takatori A, Nakamura Y, et al. Effects of novel small compounds targeting TrkB on neuronal cell survival and depression-like behavior. Neurochem Int. 2016;97:42-48.
65. Morale MC, Serra PA, L'Episcopo F, et al. Estrogen, neuroinflammation and neuroprotection in Parkinson's disease: glia dictates resistance versus vulnerability to neurodegeneration. Neuroscience. 2006;138:869-878.

66. Shea TB, Ortiz D. 17 Beta-estradiol alleviates synergistic oxidative stress resulting from folate deprivation and amyloid-beta treatment. J Alzheimers Dis. 2003;5:323-327.

67. Zhao L, Mao Z, Schneider LS, Brinton RD. Estrogen receptor beta-selective phytoestrogenic formulation prevents physical and neurological changes in a preclinical model of human menopause. Menopause. 2011;18:1131-142.

68. Nagata K, Nakashima-Kamimura N, Mikami T, Ohsawa I, Ohta S. Consumption of molecular hydrogen prevents the stress-induced impairments in hippocampus-dependent learning tasks during chronic physical restraint in mice. Neuropsychopharmacology. 2009;34:501-508.

69. Hollands C, Tobin MK, Hsu M, et al. Depletion of adult neurogenesis exacerbates cognitive deficits in Alzheimer's disease by compromising hippocampal inhibition. Mol Neurodegener. 2017;12:64.

70. Radad K, Moldzio R, Al-Shraim M, Kranner B, Krewenka C, Rausch WD. Recent advances on the role of neurogenesis in the adult brain: therapeutic potential in Parkinson's and Alzheimer's diseases. CNS Neurol Disord Drug Targets. 2017;16:740-748.

71. Li J, Wang C, Zhang JH, Cai JM, Cao YP, Sun XJ. Hydrogenrich saline improves memory function in a rat model of amyloidbeta-induced Alzheimer's disease by reduction of oxidative stress. Brain Res. 2010;1328:152-161.

72. Gu Y, Huang CS, Inoue T, et al. Drinking hydrogen water ameliorated cognitive impairment in senescence-accelerated mice. J Clin Biochem Nutr. 2010;46:269-276.

73. Nishimaki K, Asada T, Ohsawa I, et al. Effects of molecular hydrogen assessed by an animal model and a randomized clinical study on mild cognitive impairment. Curr Alzheimer Res. 2018;15:482492.

74. Yue JK, Robinson CK, Burke JF, et al. Apolipoprotein E epsilon 4 (APOE- $\varepsilon 4$ ) genotype is associated with decreased 6-month verbal memory performance after mild traumatic brain injury. Brain Behav. 2017;7:e00791.

75. Barnes LL, Wilson RS, Bienias JL, Schneider JA, Evans DA, Bennett DA. Sex differences in the clinical manifestations of Alzheimer disease pathology. Arch Gen Psychiatry. 2005;62:685-691.

76. Shumaker SA, Legault C, Rapp SR, et al. Estrogen plus progestin and the incidence of dementia and mild cognitive impairment in postmenopausal women: the Women's Health Initiative Memory Study: a randomized controlled trial. JAMA. 2003;289:2651-2662.

77. Bai H, Yang B, Yu W, Xiao Y, Yu D, Zhang Q. Cathepsin B links oxidative stress to the activation of NLRP3 inflammasome. Exp Cell Res. 2018;362:180-187.

Received: 2018-09-20

Accepted: 2018-10-26 